\title{
Boundedness of solutions for a class of second-order differential equation with singularity
}

\section{Shunjun Jiang*}

\section{"Correspondence:}

jiangshunjun@yahoo.com.cn College of Sciences, Nanjing

University of Technology, Nanjing,

210009, People's Republic of China

\author{
Abstract \\ In this paper, we study the following second-order periodic system:

$$
x^{\prime \prime}+V^{\prime}(x)+p(t)|x|^{\alpha}=0
$$ \\ where $V(x)$ has a singularity. Under some assumptions on the $V(x)$ and $p(t)$ by Ortega's \\ small twist theorem, we obtain the existence of quasi-periodic solutions and \\ boundedness of all the solutions.
}

Keywords: boundedness of solutions; singularity; small twist theorem

\section{Introduction and main result}

In 1991, Levi [1] considered the following equation:

$$
x^{\prime \prime}+V^{\prime}(x, t)=0,
$$

where $V(x, t)$ satisfies some growth conditions and $V(x, t)=V(x, t+1)$. The author reduced the system to a normal form and then applied Moser twist theorem to prove the existence of quasi-periodic solution and the boundedness of all solutions. This result relies on the fact that the nonlinearity $V(x, t)$ can guarantee the twist condition of KAM theorem. Later, several authors improved the Levi's result; we refer to [2-4] and the references therein.

Recently, Capietto, Dambrosio and Liu [5] studied the following equation:

$$
x^{\prime \prime}+V^{\prime}(x)=F(x, t)
$$

with $F(x, t)=p(t)$ is a $\pi$-periodic function and $V(x)=\frac{1}{2} x_{+}^{2}+\frac{1}{\left(1-x^{2}\right)^{v}}-1$, where $x_{+}=$ $\max \{x, 0\}, x_{-}=\max \{-x, 0\}$ and $v$ is a positive integer. Under the Lazer-Leach assumption that

$$
1+\frac{1}{2} \int_{0}^{\pi} p\left(t_{0}+\theta\right) \sin \theta d \theta>0, \quad \forall t_{0} \in R
$$

they prove the boundedness of solutions and the existence of quasi-periodic solution by KAM theorem. It is the first time that the equation of the boundedness of all solution is treated in case of a singular potential.

\section{祭 Springer}

(c) 2013 Jiang; licensee Springer. This is an Open Access article distributed under the terms of the Creative Commons Attribution License (http://creativecommons.org/licenses/by/2.0), which permits unrestricted use, distribution, and reproduction in any medium, provided the original work is properly cited. 
We observe that $F(x, t)=p(t)$ in (1.2) is smooth and bounded, so a natural question is to find sufficient conditions on $F(x, t)$ such that all solutions of (1.2) are bounded when $F(x, t)$ is unbounded. The purpose of this paper is to deal with this problem.

Motivated by the papers $[1,5,6]$, we consider the following equation:

$$
x^{\prime \prime}+V^{\prime}(x)+p(t)|x|^{\alpha}=0,
$$

where $p(t)$ is a $\pi$-periodic function,

$$
V(x)=\frac{1}{2} x_{+}^{2}+\frac{1}{1-x_{-}^{2}}-1, \quad 0<\alpha<1, x>-1 .
$$

We suppose Lazer-Leach assumption hold:

$$
\int_{0}^{\pi} p\left(t_{0}+\theta\right)(\sin \theta)^{1+\alpha} d \theta>0, \quad \forall t_{0} \in R
$$

Our main result is the following theorem.

Theorem 1 Under the assumptions (1.5) and (1.6), all the solutions of (1.4) are bounded.

The main idea of our proof is acquired from [6]. The proof of Theorem 1 is based on a small twist theorem due to Ortega [7]. It mainly consists of two steps. The first one is to transform (1.4) into a perturbation of integrable Hamilton system. The second one is to show that Poincaré map of the equivalent system satisfies Ortega's twist theorem, then some desired result can be obtained.

Moreover, we have the following theorem on solutions of Aubry-Mather type.

Theorem 2 Assume that $p(t) \in C(R)$ satisfies (1.6); then, there is an $\epsilon_{0}>0$ such that, for any $\omega \in\left(\frac{1}{\pi}, \frac{1}{\pi}+\epsilon_{0}\right)$, $t$ equation (1.4) has a solution $\left(x_{\omega}(t), x_{\omega}^{\prime}(t)\right)$ of the Mather type with rotation number $\omega$. More precisely:

Case 1: $\omega=\frac{p}{q}$ is rational. The solutions $\left(x_{\omega}(t+2 i \pi), x_{\omega}^{\prime}(t+2 i \pi)\right), 1 \leq i \leq q-1$ are independent periodic solutions of periodic $q \pi$; moreover, in this case,

$$
\lim _{q \rightarrow \infty} \min _{t \in R}\left(\left|x_{\omega}(t)\right|+\left|x_{\omega}^{\prime}(t)\right|\right)=+\infty
$$

Case 2: $\omega$ is irrational. The solution $\left(x_{\omega}(t), x_{\omega}^{\prime}(t)\right)$ is either a usual quasi-periodic solution or a generalized one.

We will apply Aubry-Mather theory, more precisely, the theorem in [8], to prove this theorem.

\section{Proof of theorem}

\subsection{Action-angle variables and some estimates}

Observe that (1.4) is equivalent to the following Hamiltonian system:

$$
x^{\prime}=\frac{\partial H}{\partial y}, \quad y^{\prime}=-\frac{\partial H}{\partial x}
$$


with the Hamiltonian function

$$
H(x, y, t)=\frac{1}{2} y^{2}+V(x)+\frac{p(t)}{(\alpha+1)}|x|^{\alpha} x .
$$

In order to introduce action and angle variables, we first consider the auxiliary autonomous equation:

$$
x^{\prime}=y, \quad y^{\prime}=-V^{\prime}(x),
$$

which is an integrable Hamiltonian system with Hamiltonian function

$$
H_{1}(x, y, t)=\frac{1}{2} y^{2}+V(x) .
$$

The closed curves $H_{1}(x, y, t)=h>0$ are just the integral curves of (2.2).

Denote by $T_{0}(h)$ the time period of the integral curve $\Gamma_{h}$ of $(2.2)$ defined by $H_{1}(x, y, t)=h$ and by $I$ the area enclosed by the closed curve $\Gamma_{h}$ for every $h>0$. Let $-1<-\alpha_{h}<0<\beta_{h}$ be such that $V\left(-\alpha_{h}\right)=V\left(\beta_{h}\right)=h$. It is easy to see that

$$
I_{0}(h)=2 \int_{-\alpha_{h}}^{\beta_{h}} \sqrt{2(h-V(s))} d s, \quad \forall h>0
$$

and

$$
T_{0}(h)=I_{0}^{\prime}(h)=2 \int_{\alpha_{h}}^{\beta_{h}} \frac{1}{\sqrt{2(h-V(s))}} d s, \quad \forall h>0 .
$$

By direct computation, we get

$$
\begin{aligned}
I_{0}(h) & =2 \int_{0}^{\beta_{h}} \sqrt{2(h-V(s))} d s+2 \int_{-\alpha_{h}}^{0} \sqrt{2(h-V(s))} d s \\
& =\pi h+2 \int_{0}^{\alpha_{h}} \sqrt{2(h-V(-s))} d s,
\end{aligned}
$$

so

$$
T_{0}(h)=\pi+\int_{0}^{\alpha_{h}} \frac{1}{\sqrt{2(h-V(-s))}} d s .
$$

We then have

$$
I_{0}(h)=I_{-}(h)+I_{+}(h), \quad T_{0}(h)=T_{-}(h)+T_{+}(h),
$$

where

$$
\begin{array}{ll}
I_{-}(h)=2 \int_{0}^{-\alpha_{h}} \sqrt{2(h-V(s))} d s, & I_{+}(h)=\pi h, \\
T_{-}(h)=2 \int_{0}^{-\alpha_{h}} \frac{1}{\sqrt{2(h-V(-s))}} d s, & T_{+}(h)=\pi .
\end{array}
$$

Similar in estimating in [5], we have the estimation of functions $I_{-}$and $T_{-}$. 
Lemma 1 We have

$$
h^{n}\left|\frac{d^{n} T_{-}(h)}{d h^{n}}\right| \leq C h^{-\frac{1}{2}}
$$

and

$$
h^{n}\left|\frac{d^{n} I_{-}(h)}{d h^{n}}\right| \leq C h^{\frac{1}{2}}
$$

where $n=0,1, \ldots, 6, h \rightarrow+\infty$. Note that here and below we always use $C, C_{0}$ or $C_{0}^{\prime}$ to indicate some constants.

Remark 1 It follows from the definitions of $T_{+}(h), T_{-}(h)$ and Lemma 1 that

$$
\lim _{h \rightarrow+\infty} T_{-}(h)=0, \quad \lim _{h \rightarrow+\infty} T_{+}(h)=\pi .
$$

Thus the time period $T_{0}(h)$ is dominated by $T_{+}(h)$ when $h$ is sufficiently large. From the relation between $T_{-}(h)$ and $I_{-}(h)$, we know $I_{0}(h)$ is dominated by $I_{+}(h)$ when $h$ is sufficiently large.

Remark 2 It also follow from the definition of $I(h), I_{-}(h), I_{+}(h)$ and Remark 1 that

$$
\left|h^{n} \frac{d^{n} I_{0}(h)}{d h^{n}}\right| \leq C_{0} I_{0}(h) \quad \text { for } n \geq 1 .
$$

Remark 3 Note that $h=h_{0}\left(I_{0}\right)$ is the inverse function of $I_{0}$. By Remark 2, we have

$$
\left|I^{n} \frac{d^{n} h(I)}{d I^{n}}\right| \leq C_{0} h(I) \quad \text { for } n \geq 1
$$

We now carry out the standard reduction to the action-angle variables. For this purpose, we define the generating function $S(x, I)=\int_{C} \sqrt{2(h-V(s))} d s$, where $C$ is the part of the closed curve $\Gamma_{h}$ connecting the point on the $y$-axis and point $(x, y)$.

We define the well-know map $(\theta, I) \rightarrow(x, y)$ by

$$
y=\frac{\partial S}{\partial x}(x, I), \quad \theta=\frac{\partial S}{\partial I}(x, I),
$$

which is symplectic since

$$
\begin{aligned}
& d x \wedge d y=d x \wedge\left(S_{x x} d x+S_{x I} d I\right)=S_{x I} d x \wedge d I, \\
& d \theta \wedge d I=\left(S_{I x} d x+S_{I I} d I\right) \wedge d I=S_{I x} d \wedge d I .
\end{aligned}
$$

From the above discussion, we can easily get

$$
\theta= \begin{cases}\frac{\pi}{T_{0}(h(x, y))}\left(\frac{T_{-}(h(x, y))}{2}+\arcsin \frac{x}{\sqrt{2(h(x, y))}}\right), & \text { if } x>0, y>0, \\ \frac{\pi}{T_{0}(h(x, y))}\left(\frac{T_{-}(h(x, y))}{2}+\pi+\arcsin \frac{x}{\sqrt{2(h(x, y))}}\right), & \text { if } x>0, y<0, \\ \frac{\pi}{T_{0}(h(x, y))}\left(\int_{-\alpha_{h}}^{x} \frac{1}{\sqrt{2\left(h(x, y)+1-\left(1-s^{2}\right)^{-1}\right)}} d s\right), & \text { if } x<0, y>0, \\ \frac{\pi}{T_{0}(h(x, y))}\left(T_{0}(h(x, y))-\int_{-\alpha_{h}}^{x} \frac{1}{\sqrt{2\left(h(x, y)+1-\left(1-s^{2}\right)^{-1}\right)}} d s\right), & \text { if } x<0, y<0\end{cases}
$$


and

$$
I(x, y)=I_{0}(h(x, y))=2 \int_{-\alpha_{h}}^{\beta_{h}} \sqrt{2(h(x, y)-V(s))} d s .
$$

In the new variables $(\theta, I)$, the system $(2.1)$ is

$$
\theta^{\prime}=\frac{\partial H}{\partial I}, \quad I^{\prime}=-\frac{\partial H}{\partial \theta}
$$

where

$$
H(\theta, I, t)=\pi h_{0}(I)+\pi \frac{p(t)}{(\alpha+1)}|x(I, \theta)|^{\alpha} x(I, \theta)
$$

In order to estimate $\pi \frac{p(t)}{(\alpha+1)}|x(I, \theta)|^{\alpha} x(I, \theta)$, we need the following lemma.

Lemma 2 [5, Lemma 2.2] For I sufficient large and $-\alpha_{h} \leq x<0$, the following estimates hold:

$$
\left|I^{n} \frac{\partial^{n} x(I, \theta)}{\partial I^{n}}\right| \leq c \sqrt{I}, \quad\left|I^{n} \frac{\partial^{n} y(I, \theta)}{\partial I^{n}}\right| \leq c \sqrt{I} \quad \text { for } 0 \leq n \leq 6 .
$$

\subsection{New action and angle variables}

Now we are concerned with the Hamiltonian system (2.5) with Hamiltonian function $H(\theta, I, t)$ given by $(2.6)$. Note that

$$
I d \theta-H d t=-(H d t-I d \theta) .
$$

This means that if one can solve $I$ from (2.6) as a function of $H$ ( $\theta$ and $t$ as parameters), then

$$
\frac{d H}{d \theta}=-\frac{\partial I}{\partial t}(t, H, \theta), \quad \frac{d t}{d \theta}=\frac{\partial I}{\partial H}(t, H, \theta)
$$

is also a Hamiltonian system with Hamiltonian function $I$ and now the action, angle and time variables are $H, t$ and $\theta$.

From (2.6) and Lemma 1, we have

$$
\frac{\partial H}{\partial I} \rightarrow 1 \quad \text { as } I \rightarrow+\infty
$$

So, we assume that $I$ can be written as

$$
I=I_{0}\left(\frac{H}{\pi}+R(H, t, \theta)\right)
$$

where $R$ satisfies $|R|<\frac{H}{\pi}$. Recalling that $h_{0}$ is the inverse function of $I_{0}$, we have

$$
\frac{H}{\pi}+R(H, t, \theta)=h_{0}(I)
$$


which implies that

$$
R(H, t, \theta)=\frac{p(t)}{(\alpha+1)}|x(I, \theta)|^{\alpha} x(I, \theta) .
$$

As a consequence, $R$ is implicitly defined by

$$
R(H, t, \theta)=\frac{p(t)}{(\alpha+1)}|x|^{\alpha} x\left(I_{0}\left(\frac{H}{\pi}+R(H, t, \theta)\right), \theta\right) .
$$

Lemma 3 The function $R(H, t, \theta)$ satisfies the following estimates:

$$
\left|\frac{\partial^{m} R(H, t, \theta)}{\partial H^{m}}\right| \leq H^{\frac{\alpha+1}{2}} \quad \text { for } m+l \leq 6
$$

Proof Case $m=0$. By (2.8), Lemma 2 and noticing that $\frac{H}{I} \rightarrow 1$ as $I \rightarrow+\infty$, we have

$$
\begin{aligned}
|R(H, t, \theta)| & =\left|\pi \frac{p(t)}{(\alpha+1)} x^{\alpha+1}\left(I_{0}\left(\frac{H}{\pi}+R(H, t, \theta)\right), \theta\right)\right| \\
& \leq\left|I_{0}\left(\frac{H}{\pi}+R(H, t, \theta)\right)\right|^{\frac{1+\alpha}{2}} p(t) \\
& =\left|I^{\frac{1+\alpha}{2}}\right| p(t) \leq C \cdot H^{\frac{1+\alpha}{2}} .
\end{aligned}
$$

Case $m \geq 1$. Derivative both sides of (2.8) with respect to $H$, we have

$$
\frac{\partial R}{\partial H}=\frac{1}{\pi} \frac{1}{-1+\frac{1}{\frac{\partial x}{\partial} I_{0}^{\prime}\left(\frac{H}{\pi}+R\right) p(t)}} .
$$

By Remark 2, Lemma 2 and the estimate of $R$, we have

$$
\left|\frac{\partial x}{\partial I} I_{0}^{\prime}\left(\frac{H}{\pi}+R\right) p(t)\right| \leq C \cdot H^{\frac{-1+\alpha}{2}} .
$$

Since

$$
\left|\frac{1}{\frac{\partial x}{\partial I} I_{0}^{\prime}\left(\frac{H}{\pi}+R\right) p(t)}\right| \gg 1 \quad \text { as } H \rightarrow+\infty,
$$

we have

$$
H\left|\frac{\partial R}{\partial H}\right| \leq C \cdot H^{\frac{1+\alpha}{2}} .
$$

We suppose that

$$
\left|\frac{\partial^{m} R(H, t, \theta)}{\partial H^{m}}\right| \leq H^{\frac{\alpha+1}{2}}
$$

holds where $m=k-1$. We will prove (2.9) also holds where $m=k, k \leq 6$. 
By direct calculation, we have

$$
\frac{\partial^{k} R}{\partial H^{k}}=\frac{\sum c_{n} j_{1} \cdots j_{n} \frac{\partial^{n} x}{\partial I^{n}} \frac{\partial j_{1}}{\partial H^{j 1}}\left(\frac{H}{\pi}+R\right) \cdots \frac{\partial^{j n}}{\partial H^{j n}}\left(\frac{H}{\pi}+R\right)}{1-\frac{\partial x}{\partial I} I_{0}^{\prime}\left(\frac{H}{\pi}+R\right) p(t)},
$$

where $1 \leq n \leq k, j_{1}+\cdots+j_{n}=k, 1 \leq j_{1}, \ldots, j_{n}<k$.

Since

$$
\frac{\partial^{j_{n}} I_{0}\left(\frac{H}{\pi}+R\right)}{\partial H^{j_{n}}}=I_{0}^{(n)} \cdot\left[\left(\frac{H}{\pi}+R\right)\right]^{\prime}+\cdots+I_{0}^{\prime} \cdot\left(\frac{H}{\pi}+R\right)^{(n)},
$$

by Lemma 1 and (2.9), when $j_{n} \geq 2$, we have

$$
\begin{aligned}
\left|\frac{\partial^{j_{n}} I_{0}\left(\frac{H}{\pi}+R\right)}{\partial H^{j_{n}}}\right| & \leq C \cdot\left|I_{0}^{\prime} \cdot\left(\frac{H}{\pi}+R\right)^{(n)}\right| \\
& \leq C \cdot H^{\frac{\alpha+1}{2}} \cdot H^{-j_{n}} \\
& \leq C \cdot H \cdot H^{-j_{n}} .
\end{aligned}
$$

When $j_{n}=1$, we have

$$
\left|\frac{\partial^{j_{n}} I_{0}\left(\frac{H}{\pi}+R\right)}{\partial H^{j_{n}}}\right| \leq C .
$$

By (2.11) and (2.12), we have

$$
\left|\frac{\partial^{j_{n}} I_{0}\left(\frac{H}{\pi}+R\right)}{\partial H^{j_{n}}}\right| \leq C \cdot H^{1-j_{n}}
$$

where $1 \leq j_{n}<k$.

By (2.13), we have

$$
\left|\frac{\partial^{j_{n}} I_{0}\left(\frac{H}{\pi}+R\right)}{\partial H^{j_{n}}}\right| \cdots\left|\frac{\partial^{j_{n}} I_{0}\left(\frac{H}{\pi}+R\right)}{\partial H^{j_{n}}}\right| \leq C \cdot H^{n-k} .
$$

By (2.10), (2.14) and Lemma 2, we have (2.9) holds where $m=k$. Thus, we prove Lemma 3.

Analogously, one may obtain, by a direct but cumbersome commutation, the following estimates.

Lemma 4 The function $R(H, t, \theta)$ satisfies the following estimates:

$$
\left|\frac{\partial^{m+l} R(H, t, \theta)}{\partial H^{m} \partial t^{l}}\right| \leq H^{\frac{\alpha+1}{2}} \quad \text { for } m+l \leq 6 .
$$

Moreover, by the implicit function theorem, there exists a function $R_{1}=R_{1}(t, H, \theta)$ such that

$$
R(H, t, \theta)=\frac{p(t)}{(\alpha+1)}|x(H, \theta)|^{\alpha} x(H, \theta)+R_{1}(H, t, \theta) .
$$


Since

$$
R_{1}(H, t, \theta)=R(H, t, \theta)-\frac{p(t)}{(\alpha+1)}|x(H, \theta)|^{\alpha} x(H, \theta)
$$

for $x \geq 0$, we have

$$
\begin{aligned}
\left|R_{1}(H, t, \theta)\right| & =\left|\frac{p(t)}{(\alpha+1)} x^{\alpha+1}\left(I_{0}\left(\frac{H}{\pi}+R(H, t, \theta)\right), \theta\right)-\frac{p(t)}{(\alpha+1)} x^{\alpha+1}(H, \theta)\right| \\
& =\left|\int_{0}^{1} x^{\alpha}\left(H+s\left(\pi R+I_{-}\right)\right) \cdot \frac{\partial x}{\partial H}\left(H+s\left(\pi R+I_{-}\right)\right) \cdot\left(\pi R+I_{-}\right) p(t) d s\right| .
\end{aligned}
$$

For $x<0$, we have

$$
\begin{aligned}
\left|R_{1}(H, t, \theta)\right| & =\left|\frac{p(t)}{(\alpha+1)}(-x)^{\alpha+1}\left(I_{0}\left(\frac{H}{\pi}+R(H, t, \theta)\right), \theta\right)-\frac{p(t)}{(\alpha+1)}(-x)^{\alpha+1}(H, \theta)\right| \\
& =\left|\int_{0}^{1}(-x)^{\alpha}\left(H+s\left(\pi R+I_{-}\right)\right) \cdot \frac{\partial x}{\partial H}\left(H+s\left(\pi R+I_{-}\right)\right) \cdot\left(\pi R+I_{-}\right) p(t) d s\right| .
\end{aligned}
$$

By Lemmas 1 and 4, we have the estimates on $R_{1}(H, t, \theta)$.

For concision, in the estimates and the calculation below, we only consider the case $x \geq 0$, since the case $x<0$ have the similar result.

Lemma $5\left|\frac{\partial^{k+l} R_{1}(H, t, \theta)}{\partial^{k} H \partial^{l} t}\right|<H^{\frac{\alpha}{2}}$ for $k+l \leq 6$.

For the estimates of $I\left(\frac{H}{\pi}+R\right)$, we need the estimates on $I_{-}\left(\frac{H}{\pi}+R\right)$. By Lemmas 1 and 5 , noticing that $|R|<\frac{H}{\pi}$, we have the following lemma.

Lemma $6\left|\frac{\partial^{k+l} I_{-}\left(\frac{H}{\pi}+R\right)}{\partial^{k} H \partial^{l} t}\right|<H^{\frac{1}{2}}$ for $k+l \leq 6$.

Now the new Hamiltonian function $I=I(t, H, \theta)$ is written in the form

$$
\begin{aligned}
I & =I_{0}\left(\frac{H}{\pi}+R\right)=I_{+}\left(\frac{H}{\pi}+R\right)+I_{-}\left(\frac{H}{\pi}+R\right) \\
& =H+\pi R(H, t, \theta)+I_{-}\left(\frac{H}{\pi}+R\right) \\
& =H+\pi \frac{p(t)}{(\alpha+1)}|x(H, \theta)|^{\alpha} x(H, \theta)+R_{1}(H, t, \theta)+I_{-}\left(\frac{H}{\pi}+R\right) .
\end{aligned}
$$

The system (2.7) is of the form

$$
\left\{\begin{array}{l}
\frac{d t}{d \theta}=\frac{\partial I}{\partial H}=1+\pi \frac{\partial x}{\partial H}(H, \theta)|x(H, \theta)|^{\alpha} p(t)+\frac{\partial R_{1}}{\partial H}(H, t, \theta)+\frac{\partial I_{-}}{\partial H}(H, t, \theta), \\
\frac{d H}{d \theta}=-\frac{\partial I}{\partial t}=-\pi \frac{p^{\prime}(t)}{(\alpha+1)}|x(\theta, H)|^{\alpha} x(\theta, H)-\frac{\partial R_{1}}{\partial t}(t, H, \theta)-\frac{\partial I_{-}}{\partial t}(H, t, \theta) .
\end{array}\right.
$$

Introduce a new action variable $\rho \in[1,2]$ and a parameter $\epsilon>0$ by $H=\epsilon^{-2} \rho$. Then $H \gg$ $1 \Leftrightarrow 0<\epsilon \ll 1$. Under this transformation, the system (2.15) is changed into the form

$$
\left\{\begin{array}{l}
\frac{d t}{d \theta}=\frac{\partial I}{\partial H}=1+\pi \frac{\partial x}{\partial H}(H, \theta)|x|^{\alpha}(H, \theta) p(t)+\frac{\partial R_{1}}{\partial H}(H, t, \theta)+\frac{\partial I_{-}}{\partial H}(H, t, \theta), \\
\frac{d \rho}{d \theta}=-\frac{\partial I}{\partial t}=-\epsilon^{2}\left[\pi \frac{p^{\prime}(t)}{(\alpha+1)}|x(\theta, H)|^{\alpha} x(\theta, H)+\frac{\partial R_{1}}{\partial t}(t, H, \theta)+\frac{\partial I_{-}}{\partial t}(H, t, \theta)\right],
\end{array}\right.
$$


which is also Hamiltonian system with the new Hamiltonian function

$$
\begin{aligned}
\Gamma(t, \rho, \theta ; \epsilon)= & \rho+\pi \epsilon^{-2} \frac{p(t)}{\alpha+1}\left|x\left(\theta, \epsilon^{-2} \rho\right)\right|^{\alpha} x\left(\theta, \epsilon^{-2} \rho\right) \\
& +\epsilon^{-2} R_{1}\left(t, \epsilon^{-2} \rho, \theta\right)+\epsilon^{-2} I_{-}\left(t, \epsilon^{-2} \rho, \theta\right) .
\end{aligned}
$$

Obviously, if $\epsilon \ll 1$, the solution $\left(t\left(\theta, t_{0}, \rho_{0}\right), \rho\left(\theta, t_{0}, \rho_{0}\right)\right)$ of (2.16) with the initial date $\left(t_{0}, \rho_{0}\right) \in R \times[1,2]$ is defined in the interval $\theta \in[0,2 \pi]$ and $\rho\left(\theta, t_{0}, \rho_{0}\right) \in\left[\frac{1}{2}, 3\right]$. So the Poincaré map of (2.16) is well defined in the domain $R \times[1,2]$.

Lemma 7 [6, Lemma 5.1] The Poincaré map of (2.16) has intersection property.

The proof is similar to the corresponding one in [6].

For convenience, we introduce the notation $O_{k}(1)$ and $o_{k}(1)$. We say a function $f(t, \rho, \theta$, $\epsilon) \in O_{k}(1)$ if $f$ is smooth in $(t, \rho)$ and for $k_{1}+k_{2} \leq k$,

$$
\left|\frac{\partial^{k_{1}+k_{2}}}{\partial t^{k_{1}} \partial \rho^{k_{2}}} f(t, \rho, \theta, \epsilon)\right| \leq C
$$

for some constant $C>0$ which is independent of the arguments $t, \rho, \theta, \epsilon$.

Similarly, we say $f(t, \rho, \theta, \epsilon) \in o_{k}(1)$ if $f$ is smooth in $(t, \rho)$ and for $k_{1}+k_{2} \leq k$,

$$
\lim _{\epsilon \rightarrow 0}\left|\frac{\partial^{k_{1}+k_{2}}}{\partial t^{k_{1}} \partial \rho^{k_{2}}} f(t, \rho, \theta, \epsilon)\right|=0
$$

uniformly in $(t, \rho, \theta)$.

\subsection{Poincaré map and twist theorems}

We will use Ortega's small twist theorem to prove that the Poincaré map $P$ has an invariant closed curve, if $\epsilon$ is sufficiently small. Let us first recall the theorem in [7].

Lemma 8 (Ortega's theorem) Let $A=\mathbb{S}^{1} \times[a, b]$ be a finite cylinder with universal cover $\mathbb{A}=\mathbb{R} \times[a, b]$. The coordinate in $\mathbb{A}$ is denoted by $(\tau, v)$. Consider a map

$$
\bar{f}: A \rightarrow \mathbb{S} \times \mathbb{R}
$$

We assume that the map has the intersection property. Suppose that $f: A \rightarrow \mathbb{R} \times \mathbb{R}$, $\left(\tau_{0}, v_{0}\right) \rightarrow\left(\tau_{1}, v_{1}\right)$ is a lift of $\bar{f}$ and it has the form

$$
\left\{\begin{array}{l}
\tau_{1}=\tau_{0}+2 N \pi+\delta l_{1}\left(\tau_{0}, v_{0}\right)+\delta \tilde{g}_{1}\left(\tau_{0}, v_{0}\right) \\
v_{1}=v_{0}+\delta l_{2}\left(\tau_{0}, v_{0}\right)+\delta \tilde{g}_{2}\left(\tau_{0}, v_{0}\right)
\end{array}\right.
$$

where $N$ is an integer, $\delta \in(0,1)$ is a parameter. The functions $l_{1}, l_{2}, \tilde{g}_{1}$ and $\tilde{g}_{2}$ satisfy

$$
\begin{aligned}
& l_{1} \in C^{6}(A), \quad l_{1}\left(\tau_{0}, v_{0}\right)>0, \quad \frac{\partial l_{1}}{\partial v_{0}}\left(\tau_{0}, v_{0}\right)>0, \quad \forall\left(\tau_{0}, v_{0}\right) \in A, \\
& l_{2}(\cdot, \cdot), \tilde{g}_{1}(\cdot, \cdot, \epsilon), \tilde{g}_{2}(\cdot, \cdot, \epsilon) \in C^{5}(A) .
\end{aligned}
$$


In addition, we assume that there is a function $I: A \rightarrow R$ satisfying

$$
I \in C^{6}(A), \quad \frac{\partial I}{\partial v_{0}}\left(\tau_{0}, v_{0}\right)>0, \quad \forall\left(\tau_{0}, \nu_{0}\right) \in A
$$

and

$$
l_{1}\left(\tau_{0}, v_{0}\right) \cdot \frac{\partial I}{\partial \tau_{o}}\left(\tau_{0}, v_{0}\right)+l_{2}\left(\tau_{0}, v_{0}\right) \cdot \frac{\partial I}{\partial \nu_{0}}\left(\tau_{0}, v_{0}\right)=0, \quad \forall\left(\tau_{0}, \nu_{0}\right) \in A
$$

Moreover, suppose that there are two numbers $\tilde{a}$, and $\tilde{b}$ such that $a<\tilde{a}<\tilde{b}<b$ and

$$
I_{M}(a)<I_{m}(\tilde{a}) \leq I_{M}(\tilde{a})<I_{m}(\tilde{b}) \leq I_{M}(\tilde{b})<I_{m}(b),
$$

where

$$
I_{M}(r)=\max _{\rho \in S^{1}} I\left(\rho_{o}, \tau_{o}\right), \quad I_{m}(r)=\min _{\rho \in S^{1}} I\left(\rho_{o}, \tau_{o}\right) .
$$

Then there exist $\epsilon>0$ and $\Delta>0$ such that, if $\delta<\Delta$ and

$$
\left\|\tilde{g}_{1}(\cdot, \cdot, \epsilon)\right\|_{C^{5}(A)}+\left\|\tilde{g}_{2}(\cdot, \cdot, \epsilon)\right\|_{C^{5}(A)}<\epsilon,
$$

the mapping $\bar{f}$ has an invariant curve in $\Gamma_{A}$. The constant $\epsilon$ is independent of $\delta$.

We make the ansatz that the solution of (2.16) with the initial condition $(t(0), \rho(0))=$ $\left(t_{0}, \rho_{0}\right)$ is of the form

$$
t=t_{0}+\theta+\epsilon^{1-\alpha} \Sigma_{1}\left(t_{0}, \rho_{0}, \theta ; \epsilon\right), \quad \rho=\rho_{0}+\epsilon^{1-\alpha} \Sigma_{2}\left(t_{0}, \rho_{0}, \theta ; \epsilon\right) .
$$

Then the Poincaré map of $(2.16)$ is

$$
P: t_{1}=t_{0}+2 \pi+\epsilon^{1-\alpha} \Sigma_{1}\left(t_{0}, \rho_{0}, 2 \pi ; \epsilon\right), \quad \rho_{1}=\rho_{0}+\epsilon^{1-\alpha} \Sigma_{2}\left(t_{0}, \rho_{0}, 2 \pi ; \epsilon\right) .
$$

The functions $\Sigma_{1}$ and $\Sigma_{2}$ satisfy

$$
\left\{\begin{aligned}
\Sigma_{1}= & \pi \epsilon^{\alpha-1} \int_{0}^{\theta} \frac{\partial x}{\partial H}\left(\theta, \epsilon^{-2} \rho\right)|x|^{\alpha} p(t) d \theta \\
& +\epsilon^{\alpha-1} \int_{0}^{\theta}\left(\frac{\partial R_{1}}{\partial H}(H, t, \theta)+\frac{\partial I_{-}}{\partial H}(H, t, \theta)\right) d \theta, \\
\Sigma_{2}= & -\frac{\pi \epsilon^{\alpha+1}}{\alpha+1} \int_{0}^{\theta}\left|x\left(\theta, \epsilon^{-2} \rho\right)\right|^{\alpha} x\left(\theta, \epsilon^{-2} \rho\right) p^{\prime}(t) d \theta \\
& -\frac{\epsilon^{\alpha+1}}{\alpha+1} \int_{0}^{\theta}\left(\frac{\partial R_{1}}{\partial t}(H, t, \theta)-\frac{\partial I_{-}}{\partial t}(H, t, \theta)\right) d \theta,
\end{aligned}\right.
$$

where $t=t_{0}+\theta+\epsilon^{1-\alpha} \Sigma_{1}, \rho=\rho_{0}+\epsilon^{1-\alpha} \Sigma_{2}$. By Lemmas 4, 6 and 7, we know that

$$
\left|\Sigma_{1}\right|+\left|\Sigma_{2}\right| \leq C \text { for } \theta \in[0,2 \pi] .
$$

Hence, for $\rho_{0} \in[1,2]$, we may choose $\epsilon$ sufficiently small such that

$$
\rho_{0}+\epsilon \Sigma_{2} \geq \frac{\rho_{0}}{2} \geq \frac{1}{2} .
$$


Moreover, we can prove that

$$
\Sigma_{1}, \Sigma_{2} \in O_{6}(1) .
$$

Lemma 9 The following estimates hold:

$$
\begin{aligned}
& x^{\alpha+1}\left(\theta, \epsilon^{-2} \rho\right)-x^{\alpha+1}\left(\theta, \epsilon^{-2} \rho_{0}\right) \in \epsilon^{-\alpha} O_{6}(1), \\
& \frac{\partial x}{\partial H}\left(\theta, \epsilon^{-2} \rho\right) x^{\alpha}\left(\theta, \epsilon^{-2} \rho\right)-\frac{\partial x}{\partial H}\left(\theta, \epsilon^{-2} \rho_{0}\right) x^{\alpha}\left(\theta, \epsilon^{-2} \rho_{0}\right) \in \epsilon^{2-\alpha} O_{6}(1) .
\end{aligned}
$$

Proof Let

$$
\begin{aligned}
\Delta\left(t_{0}, \rho_{0}, \theta\right)= & x^{\alpha+1}\left(\theta, \epsilon^{-2} \rho\right)-x^{\alpha+1}\left(\theta, \epsilon^{-2} \rho_{0}\right) \\
= & \int_{0}^{1}(\alpha+1) x^{\alpha}\left(\theta, \epsilon^{-2} \rho_{0}+s \epsilon^{-1} \Sigma_{2}\right) \\
& \cdot \frac{\partial x}{\partial H}\left(\theta, \epsilon^{-2} \rho_{0}+s \epsilon^{-1} \Sigma_{2}\right) \epsilon^{-1} \Sigma_{2} d s .
\end{aligned}
$$

By Lemma 2 and (2.25), we have

$$
\begin{aligned}
\left|\Delta\left(t_{0}, \rho_{0}, \theta\right)\right| & \leq C \cdot\left(\epsilon^{-2} \rho_{0}+s \epsilon^{-1} \Sigma_{2}\right)^{\frac{\alpha}{2}}\left(\epsilon^{-2} \rho_{0}+s \epsilon^{-1} \Sigma_{2}\right)^{-\frac{1}{2}} \epsilon^{-1} \Sigma_{2} \\
& \leq C \cdot\left(\epsilon^{-2} \rho_{0}+s \epsilon^{-1} \Sigma_{2}\right)^{\frac{\alpha-1}{2}} \epsilon^{\alpha-1} \epsilon^{-\alpha} \\
& \leq C \cdot \epsilon^{-\alpha}
\end{aligned}
$$

Take the derivative with respect to $\rho_{0}$ in the both sides of $\Delta\left(t_{0}, \rho_{0}, \theta\right)$, we have

$$
\begin{aligned}
\frac{\partial \Delta}{\partial \rho_{0}}= & \int_{0}^{1}\left[(\alpha+1) \alpha x^{\alpha-1} \frac{\partial x}{\partial H} \frac{1+s \epsilon \frac{\partial \Sigma_{2}}{\partial \rho_{0}}}{\epsilon^{2}} \frac{\partial x}{\partial H} \frac{\Sigma_{2}}{\epsilon}+(\alpha+1) x^{\alpha} \frac{\partial^{2} x}{\partial H^{2}} \frac{1+s \epsilon \frac{\partial \Sigma_{2}}{\partial \rho_{0}}}{\epsilon^{2}} \frac{\Sigma_{2}}{\epsilon}\right. \\
& \left.+(\alpha+1) x^{\alpha} \frac{\partial x}{\partial H} \frac{\partial \Sigma_{2}}{\partial \rho_{0}} \frac{1}{\epsilon}\right] d s .
\end{aligned}
$$

Using Lemma 2 and noticing $|\Delta| \leq C \cdot \epsilon^{-\alpha}$, we have

$$
\left|\frac{\partial \Delta}{\partial \rho_{0}}\right| \leq C \cdot \epsilon^{-\alpha}
$$

Analogously, one may obtain, by a direct but cumbersome commutation that

$$
\left|\frac{\partial^{k+l} \Delta}{\partial \rho_{0}^{k} \partial t_{0}^{l}}\right| \leq C \cdot \epsilon^{-\alpha}
$$

which means that

$$
x^{\alpha+1}\left(\theta, \epsilon^{-2} \rho\right)-x^{\alpha+1}\left(\theta, \epsilon^{-2} \rho_{0}\right) \in \epsilon^{-\alpha} O_{6}(1) .
$$

The estimates for $\frac{\partial x}{\partial H}\left(\theta, \epsilon^{-2} \rho\right) x^{\alpha}\left(\theta, \epsilon^{-2} \rho\right)-\frac{\partial x}{\partial H}\left(\theta, \epsilon^{-2} \rho_{0}\right) x^{\alpha}\left(\theta, \epsilon^{-2} \rho_{0}\right)$ follow from a similar argument, we omit it here. Thus, Lemma 9 is proved. 
Now we turn to give an asymptotic expression of Poincaré map of (2.15), that is, we study the behavior of the functions $\Sigma_{1}$ and $\Sigma_{2}$ at $\theta=\pi$ as $\epsilon \rightarrow 0$. In order to estimate $\Sigma_{1}$ and $\Sigma_{2}$, we need introduce the following definition and lemma. Let

$$
\Theta_{+}(I)=\operatorname{meas}\left\{\theta \in[0, \pi], x\left(H_{0}, \theta\right)>0\right\}, \quad \Theta_{-}(I)=T_{0}-\Theta_{+}(I)
$$

where $H_{0}=\epsilon^{-2} \rho_{0}$.

\section{Lemma 10}

$$
\Theta_{+}(I)=\pi+\epsilon O_{6}(1), \quad \Theta_{-}(I)=\epsilon O_{6}(1) .
$$

Proof This lemma was proved in [5], so we omit the details.

For estimate $\Sigma_{1}$ and $\Sigma_{2}$, we need the estimates of $x$ and $x_{H}$.

We recall that when $x<0$, we have

$$
\left|x\left(H_{0}, \theta\right)\right|=O_{6}(1), \quad\left|x_{H}\left(H_{0}, \theta\right)\right|=\epsilon^{2} O_{5}(1) .
$$

When $x>0$, by the definition of $\theta$, we have

$$
\arcsin \frac{x\left(H_{0}, \theta\right)}{\sqrt{2 h}}=\frac{T_{0}(h)}{\pi} \theta-\frac{T_{-}(h)}{2}=\theta+\epsilon^{2} O_{5}(1),
$$

which yields that

$$
x\left(H_{0}, \theta\right)=\sqrt{\frac{2 H_{0}}{\pi}} \sin \theta+O_{5}(1), \quad x_{H}\left(H_{0}, \theta\right)=\sqrt{\frac{1}{2 H_{0} \pi}} \sin \theta+\epsilon^{2} O_{5}(1) .
$$

Now we can give the estimates of $\Sigma_{1}$ and $\Sigma_{2}$.

Lemma 11 The following estimates hold true:

$$
\begin{aligned}
& \Sigma_{1}\left(t_{0}, \rho_{0}, 2 \pi ; \epsilon\right)=\left(\frac{\pi}{2 \rho_{0}}\right)^{\frac{\alpha-1}{2}} \int_{0}^{\pi}(\sin \theta)^{1+\alpha} p\left(t_{0}+\theta\right) d \theta+o_{6}(1), \\
& \Sigma_{2}\left(t_{0}, \rho_{0}, 2 \pi ; \epsilon\right)=-\frac{1}{\alpha+1} \pi^{\frac{1-\alpha}{2}}\left(2 \rho_{0}\right)^{\frac{\alpha+1}{2}} \int_{0}^{\pi}(\sin \theta)^{1+\alpha} p^{\prime}\left(t_{0}+\theta\right) d \theta+o_{6}(1)
\end{aligned}
$$

for $\epsilon \rightarrow 0$.

Proof Firstly, we consider $\Sigma_{1}$. By Lemmas 2, 6 and (2.23), we have

$$
\begin{aligned}
\Sigma_{1}\left(t_{0}, \rho_{0}, 2 \pi ; \epsilon\right)= & \pi \epsilon^{\alpha-1} \int_{0}^{\pi} \frac{\partial x}{\partial H}\left(\theta, \epsilon^{-2} \rho\right)\left|x\left(\theta, \epsilon^{-2} \rho\right)\right|^{\alpha} p(t) d \theta \\
& +\epsilon^{\alpha-1} \int_{0}^{\pi}\left(\frac{\partial R_{1}}{\partial H}\left(\epsilon^{-2} \rho, t, \theta\right)+\frac{\partial I_{-}}{\partial H}\left(\epsilon^{-2} \rho, t, \theta\right)\right) d \theta \\
= & \pi \epsilon^{\alpha-1} \int_{0}^{\pi} \frac{\partial x}{\partial H}\left(\theta, \epsilon^{-2} \rho_{0}\right)\left|x\left(\theta, \epsilon^{-2} \rho_{0}\right)\right|^{\alpha} p\left(t_{0}+\theta\right) d \theta+\epsilon^{\alpha} O_{6}(1)
\end{aligned}
$$




$$
\begin{aligned}
= & \pi \epsilon^{\alpha-1}\left(\int_{\Theta_{+}} \frac{\partial x}{\partial H}\left(\theta, \epsilon^{-2} \rho\right)|x|^{\alpha} p\left(t_{0}+\theta\right) d \theta\right. \\
& \left.+\int_{\Theta_{-}} \frac{\partial x}{\partial H}\left(\theta, \epsilon^{-2} \rho\right)|x|^{\alpha} p\left(t_{0}+\theta\right) d \theta\right)+\epsilon^{\alpha} O_{6}(1) \\
= & \pi \epsilon^{\alpha-1} \int_{\Theta_{+}} \frac{\partial x}{\partial H}\left(\theta, \epsilon^{-2} \rho\right)|x|^{\alpha} p\left(t_{0}+\theta\right) d \theta+\epsilon^{\alpha} O_{6}(1) \\
= & \pi \epsilon^{\alpha-1} \int_{0}^{\pi} \frac{\partial x}{\partial H}\left(\theta, \epsilon^{-2} \rho\right)|x|^{\alpha} p\left(t_{0}+\theta\right) d \theta+\epsilon^{\alpha} O_{6}(1) \\
= & \left(\frac{\pi}{2 \rho_{0}}\right)^{\frac{1-\alpha}{2}} \int_{0}^{\pi}(\sin \theta)^{\alpha+1} p\left(t_{0}+\theta\right) d \theta+o_{6}(1) .
\end{aligned}
$$

Now we consider $\Sigma_{2}$.

$$
\begin{aligned}
\Sigma_{2}\left(t_{0}, \rho_{0}, 2 \pi ; \epsilon\right)= & -\frac{\pi \epsilon^{\alpha+1}}{\alpha+1} \int_{0}^{\pi}\left|x\left(\theta, \epsilon^{-2} \rho\right)\right|^{\alpha} x\left(\theta, \epsilon^{-2} \rho\right) p^{\prime}(t) d \theta \\
& -\frac{\epsilon^{\alpha+1}}{\alpha+1} \int_{0}^{\pi}\left(\frac{\partial R_{1}}{\partial t}\left(\epsilon^{-2} \rho, t, \theta\right)+\frac{\partial I_{-}}{\partial t}\left(\epsilon^{-2} \rho, t, \theta\right)\right) d \theta \\
= & -\frac{\pi \epsilon^{\alpha+1}}{\alpha+1} \int_{0}^{\pi}\left|x\left(\theta, \epsilon^{-2} \rho_{0}\right)\right|^{\alpha} x\left(\theta, \epsilon^{-2} \rho_{0}\right) p^{\prime}\left(t_{0}+\theta\right) d \theta+\epsilon^{\alpha} O_{6}(1) \\
= & -\frac{\pi \epsilon^{\alpha+1}}{\alpha+1}\left(\int_{\Theta_{+}}\left|x\left(\theta, \epsilon^{-2} \rho\right)\right|^{\alpha} x\left(\theta, \epsilon^{-2} \rho_{0}\right) p^{\prime}\left(t_{0}+\theta\right) d \theta\right. \\
& \left.+\iint_{\Theta-}\left|x\left(\theta, \epsilon^{-2} \rho_{0}\right)\right|^{\alpha} x\left(\theta, \epsilon^{-2} \rho\right) p^{\prime}\left(t_{0}+\theta\right) d \theta\right)+\epsilon^{\alpha} O_{6}(1) \\
= & -\frac{\pi \epsilon^{\alpha+1}}{\alpha+1} \int_{\Theta}\left|x\left(\theta, \epsilon^{-2} \rho\right)\right|^{\alpha} x\left(\theta, \epsilon^{-2} \rho_{0}\right) p^{\prime}\left(t_{0}+\theta\right) d \theta+\epsilon^{\alpha} O_{6}(1) \\
= & -\frac{\pi \epsilon^{\alpha+1}}{\alpha+1} \int_{0}^{\pi}\left|x\left(\theta, \epsilon^{-2} \rho_{0}\right)\right|^{\alpha} x\left(\theta, \epsilon^{-2} \rho\right) p^{\prime}\left(t_{0}+\theta\right) d \theta+\epsilon^{\alpha} O_{6}(1) \\
= & -\frac{1}{\alpha+1} \pi \frac{1-\alpha}{2}\left(2 \rho_{0}\right)^{\frac{\alpha+1}{2}} \int_{0}^{\pi}(\sin \theta)^{1+\alpha} p^{\prime}\left(t_{0}+\theta\right) d \theta+o_{6}(1) .
\end{aligned}
$$

Thus, Lemma 11 is proved.

\subsection{Proof of Theorem 1}

Let

$$
\begin{aligned}
& \Psi_{1}\left(t_{0}, \rho_{0}\right)=\left(\frac{\pi}{2 \rho_{0}}\right)^{\frac{1-\alpha}{2}} \int_{0}^{\pi}(\sin \theta)^{1+\alpha} p\left(t_{0}+\theta\right) d \theta \\
& \Psi_{2}\left(t_{0}, \rho_{0}\right)=-\frac{1}{\alpha+1} \pi^{\frac{1-\alpha}{2}}\left(2 \rho_{0}\right)^{\frac{\alpha+1}{2}} \int_{0}^{\pi}(\sin \theta)^{1+\alpha} p^{\prime}\left(t_{0}+\theta\right) d \theta .
\end{aligned}
$$

Then there are two functions $\phi_{1}$ and $\phi_{2}$ such that the Poincaré map of (2.16), given by (2.22), is of the form

$$
P: t_{1}=t_{0}+2 \pi+\epsilon^{1-\alpha} \Psi_{1}\left(t_{0}, \rho_{0}\right)+\epsilon^{1-\alpha} \phi_{1}, \quad \rho_{1}=\rho_{0}+\epsilon^{1-\alpha} \Psi_{2}\left(t_{0}, \rho_{0}\right)+\epsilon^{1-\alpha} \phi_{2},
$$

where $\phi_{1}, \phi_{2} \in o_{6}(1)$. 
Since $\int_{0}^{\pi} p\left(t_{0}+\theta\right) \sin \theta d \theta>0, \forall t_{0} \in R$, we have

$$
\Psi_{1}>0, \quad \frac{\partial \Psi_{1}}{\partial \rho_{0}} \neq 0 .
$$

Let

$$
L=\frac{\rho_{0}^{-\frac{\alpha+1}{2}}}{\int_{0}^{\pi}(\sin \theta)^{1+\alpha} p\left(t_{0}+\theta\right) d \theta} .
$$

Then

$$
\frac{\partial L}{\partial t_{0}} \Psi_{1}\left(t_{0}, \rho_{0}\right)+\frac{\partial L}{\partial \rho_{0}} \Psi_{2}\left(t_{0}, \rho_{0}\right)=0 .
$$

The other assumptions of Ortega's theorem are easily verified. Hence, there is an invariant curve of $P$ in the annulus $\left(t_{0}, \rho_{0}\right) \in S^{1} \times[1,2]$ which imply that the boundedness of our original equation (1.4). Then Theorem 1 is proved.

\subsection{Proof of Theorem 2}

We apply Aubry-Mather theory. By Theorem B in [8] and the monotone twist property of the Poincaré map $P$ guaranteed by $\frac{\partial \Psi_{1}}{\partial \rho_{0}}<0$. It is straightforward to check that Theorem 2 is correct.

Remark 4 In [9], the authors study the multiplicity of positive periodic solutions of singular Duffing equations

$$
x^{\prime \prime}+g(x)=p(t)
$$

where $g(x)$ satisfies the semilinear condition at infinity and the time map satisfies an oscillation condition, and prove that the given equation possesses infinitely many positive $2 \pi$-periodic solutions by using the Poincaré-Birkhoff theorem. By the methods and techniques in [9], we can also prove the existence of $2 \pi$-periodic solutions of (1.4) where $V(x)$ satisfies the sublinear condition.

\section{Competing interests}

The author declares that they have no competing interests.

\section{Acknowledgements}

Thanks are given to referees whose comments and suggestions were very helpful for revising our paper.

Received: 14 September 2012 Accepted: 22 March 2013 Published: 10 April 2013

\section{References}

1. Levi, M: Quasiperiodic motions in superquadratic time-periodic potential. Commun. Math. Phys. 144, 43-82 (1991)

2. Kupper, T, You, J: Existence of quasiperiodic solutions and Littlewood's boundedness problem of Duffing equations with subquadratic potentials. Nonlinear Anal. 35, 549-559 (1999)

3. Li, X: Boundedness of solutions for Duffing's equations with semilinear potentials. J. Differ. Equ. 176, 248-268 (2001)

4. Wang, Y: Boundedness for sublinear Duffing equations with time-dependent potential. J. Differ. Equ. 247, 104-118 (2009)

5. Capietto, A, Dambrosio, W, Liu, B: On the boundedness of solutions to a nonlinear singular oscillator. Z. Angew. Math. Phys. 60(6), 1007-1034 (2009)

6. Liu, B: Quasi-periodic solutions of forced isochronous oscillators at resonance. J. Differ. Equ. 246, 3471-3495 (2009) 
7. Ortega, R: Boundedness in a piecewise linear oscillator and a variant of the small twist theorem. Proc. Lond. Math. Soc. 79, 381-413 (1999)

8. Pei, ML: Aubry-Mather sets for finite-twist maps of a cylinder and semilinear Duffing equations. J. Differ. Equ. 113, 106-127 (1994)

9. Wang, Z, Ma, T: Existence and multiplicity of periodic solutions of semilinear resonant Duffing equations with singularities. Nonlinearity 25, 279-307 (2012)

doi:10.1186/1687-2770-2013-84

Cite this article as: Jiang: Boundedness of solutions for a class of second-order differential equation with singularity. Boundary Value Problems 2013 2013:84.

Submit your manuscript to a SpringerOpen ${ }^{\odot}$ journal and benefit from:

- Convenient online submission

- Rigorous peer review

- Immediate publication on acceptance

Open access: articles freely available online

- High visibility within the field

- Retaining the copyright to your article

Submit your next manuscript at $>$ springeropen.com 